\title{
REVISÃO DE LITERATURA SOBRE A APLICAÇÃO DA ENTREVISTA MOTIVACIONAL BREVE EM USUÁRIOS NOCIVOS E DEPENDENTES DE ÁLCOOL
}

\author{
Cristiane Martins Baía Sales* \\ Neliana Buzi Figlie
}

\begin{abstract}
RESUMO. Este artigo apresenta os elementos necessários para a compreensão do uso da Entrevista Motivacional Breve em usuários nocivos e dependentes de álcool através de uma revisão da literatura sobre sua estrutura metodológica e efetividade. $\mathrm{O}$ edstudo inclui a revisão e discussão sobre o uso desta abordagem no Brasil. Foram selecionados 18 estudos internacionais e nacionais e seus resultados foram discutidos a partir da apresentação de duas tabelas com os principais resultados. A Entrevista Motivacional Breve consiste numa abordagem eficaz para usuários nocivos e dependentes de álcool; possui uma metodologia prática e objetiva, que permite ser ela aplicada por qualquer profissional treinado, podendo ser utilizada sozinha, acoplada a outras técnicas ou mesmo como prelúdio, preparando uma base motivacional para outros tratamentos. No Brasil mais pesquisas são necessárias para que se encontrem meios eficientes, eficazes e de baixo custo para tratar indivíduos que se encontrem e qualquer dos diferentes estágios desta doença.
\end{abstract}

Palavras-chave: Entrevista Motivacional Breve; álcool; tratamento.

\section{A LITERATURE REVISION ABOUT THE APPLICATION OF THE BRIEF MOTIVATIONAL INTERVIEWING IN HARMFUL USERS AND ALCOHOL DEPENDENTS}

\begin{abstract}
This article presents the necessary elements for the understanding of the use of the Brief Motivational Interviewing (BMI) in harmful users and alcohol dependents, through a literature revision about its methodological and effectiveness structure. This essay also includes the revision and discussion about this approach use in Brazil. Eighteen national and international studies have been selected and their results have been discussed from the presentation of two tables with the main results. The BMI consists of an efficient approach for the harmful users and alcohol dependent. It has a practical and objective methodology that permits to be applied by any trained Professional and it can be used alone or with other technics, or even as an overture that prepares for a motivational basis for other treatments. In Brazil more researches are necessary in order to find efficient and low costs means to treat the patients who are in different stages of this disease.
\end{abstract}

Key words: Brief Motivational Interviewing; alcohol; treatment.

\section{UNA REVISIÓN DE LA LITERATURA SOBRE LA APLICACIÓN DE LA ENTREVISTA MOTIVACIONAL BREVE EN ABUSO O DEPENDENCIA DEL ALCOHOL}

\begin{abstract}
RESUMEN. Este artículo presenta los elementos necesarios para la comprensión del uso de la Entrevista Motivacional Breve en usuarios nocivos y dependientes de Alcohol a través de una revisión de la literatura sobre la estructura de su metodología y efectividad. Este trabajo también incluye la revisión y discusión sobre el uso de este abordaje en Brasil. Fueron seleccionados 18 estudios internacionales y nacionales y sus resultados fueron discutidos a partir de la presentación de sus tablas con los principales resultados. La Entrevista Motivacional Breve consiste en un abordaje eficaz para usuarios nocivos y dependientes del alcohol, posee metodología práctica y objetiva que permite ser aplicada por cualquier profesional entrenado, podiendo ser utilizada sola, acoplada a otras técnicas o aun como preludio preparando una base motivacional para otros tratamientos. En Brasil más pesquisas son necesarias para que se encuentren medios eficientes, eficaces y de bajo costo para tratar a los individuos que se encuentran en los diferentes estadios de esta enfermedad.
\end{abstract}

Palabras-clave: Entrevista Motivacional Breve; alcohol; tratamiento.

\footnotetext{
* Psicóloga da UNIAD (Unidade de Pesquisas em Álcool e Drogas) da UNIFESP. Especialista em Dependência Química e em Entrevista Motivacional por membros do MINT (Motivational Interviewing Network Trainers) - RJ.

\# Psicóloga, Mestre em Saúde Mental e Doutora em Ciências. Professora do Programa de Pós-Graduação em Psiquiatria.
} 
Pela extensão dos danos que acarreta, a dependência alcoólica é um dos grandes problemas atuais de saúde pública, contribuindo para a redução da saúde física e emocional do dependente e de seus familiares, além de problemas sociais e econômicos.

Um estudo epidemiológico recente estimou que $12 \%$ da população adulta brasileira fazem uso nocivo ou são dependentes de álcool (Laranjeira, Pinsky, Zaleski \& Caetano, 2007). Grande tem sido a preocupação com o aumento do consumo de álcool em todo o mundo. No Brasil, esta progressão se deve, em parte, a ser o álcool uma droga de baixo custo e de fácil acesso a todas as camadas sociais.

Além de ser pequena a porcentagem de dependentes de álcool que buscam programas de tratamento especializado, a maioria destes frequentemente comparece a outras especialidades médicas para tratar de complicações álcool-induzidas, como gastrites, depressões e traumatismos (Figlie, Pillon, Laranjeira, \& Dunn, 2000). Destarte, a identificação, domínio do conhecimento e posterior tratamento da dependência química representam um grande desafio e responsabilidade de todos os profissionais de saúde, independentemente de sua especialidade (Fleming \& Manwell, 1999).

Não há soluções simples para ajudar as pessoas a mudar um comportamento, por isso pesquisadores e profissionais da área da saúde buscam constantemente formas mais eficazes de atuar junto a seus pacientes. Nos últimos vinte anos houve uma grande evolução no conhecimento a respeito do processo de mudança em si e na descoberta de estratégias de intervenção efetivas na promoção da mudança de comportamento. Existe um amplo espectro de utilizações desses conhecimentos no tratamento do alcoolismo, devendo as intervenções ser dirigidas tanto à população que busca tratamento quanto àquela que não procura por ajuda, considerando-se que neste último grupo está a grande maioria dos dependentes de álcool e usuários nocivos.

\section{O PROBLEMA EM RELAÇÃO AO DIAGNÓSTICO DE USO NOCIVO OU DEPENDÊNCIA}

Segundo a Organização Mundial de Saúde o período médio compreendido entre o primeiro problema decorrente do uso do álcool e a primeira intervenção voltada a este problema é de cinco anos, ou seja, o alcoolismo é identificado em seus estágios finais, num quadro de dependência grave, quando há evidências de grandes perdas e reduzidas chances de reabilitação.

Diagnosticar e tratar a dependência do álcool precocemente tem uma importância fundamental no prognóstico do paciente. Cerca de um quinto dos indivíduos tratados pela rede primária de saúde ingere uma quantidade de álcool considerada de alto risco e nociva (Fleming \& Manwell, 1999). Estudos comprovam a necessidade de intervenções em dependentes químicos que buscam apenas $\mathrm{o}$ tratamento clínico não especializado, visto que a maior parte destes frequenta ambulatórios gerais, prontos-socorros ou hospitais gerais em fases iniciais da doença (Fleming \& Manwell, 1999). Diante disso, o tratamento da dependência química é um grande desafio não só para especialistas, mas para todos os profissionais da área da saúde, que no exercício de sua função devem buscar formas mais eficazes de identificar em seus pacientes problemas de uso nocivo/dependência do álcool e atuar junto a eles.

\section{A ENTREVISTA MOTIVACIONAL}

A Entrevista Motivacional (EM) é uma abordagem psicológica desenvolvida por Miller e Rollnick (1991) e difundida na Europa, nos EUA e, há quase uma década, no Brasil. Foi fundamentada em elementos de outras técnicas já existentes, tais como a terapia centrada no cliente e terapias breves, acrescentando alguns novos conceitos para cumprir o seu principal objetivo: promover a mudança de comportamento.

Os conceitos de motivação, ambivalência e prontidão para a mudança, essenciais à compreensão das dependências, são os pressupostos norteadores desta abordagem. A EM surgiu a partir de experiências clínicas com dependentes de álcool e atualmente sua eficácia é consagrada para o beber problemático (Vasilaki, Hosier \& Cox, 2006) e outras dependências químicas (Dunn, Deroo \& Rivara, 2001; Hettema, Steele \& Miller, 2005), bem como no tratamento de doenças como hipertensão, diabetes, comorbidades psiquiátricas e transtornos alimentares (Hettema, Steele \& Miller, 2005; Rubak, Sandboek, Lauritzen \& Christensen, 2005;).

Expressar empatia, desenvolver a discrepância entre o comportamento presente e as metas do cliente, evitar argumentação, acompanhar a resistência e promover a autoeficácia são os cinco princípios da EM que orientam a conduta do terapeuta em todo contato com o cliente, em qualquer setting ou fase do tratamento.

\section{ENTREVISTA MOTIVACIONAL BREVE}

A abordagem da motivação foi introduzida na estrutura da Intervenção Breve (IB) em função não só da brevidade da técnica, mas principalmente da observação 
de que a comunicação empática voltada para a prontidão para mudança do cliente era tão importante quanto o diagnóstico em si. Esse modelo considera a mudança comportamental como um processo e leva em conta que as pessoas passam por diferentes estágios motivacionais. O modelo teórico tem início com a Pré-Contemplação, estágio em que a pessoa não considera a necessidade de ajuda, não demonstrando consciência suficiente de que tem problemas, embora outras pessoas do seu convívio possam estar cientes disso. Quando a pessoa visualiza seu consumo de substâncias como um problema com possibilidade de mudanças, ela entra no estágio de Contemplação. Seguindo para o estágio de Preparação, a pessoa começa a pensar em tentativas para mudar seu comportamento. Neste estágio, geralmente as pessoas são descritas como prontas para a ação. Quando estas tentativas são colocadas em prática surge o estágio de ação, ocorrendo uma implementação de planos para a modificação do comportamento aditivo envolvendo tentativas concretas de modificar comportamentos, experiências e/ou o meio ambiente, a fim de superar os problemas da dependência. Finalmente, o sucesso da mudança ocorre no estágio de manutenção, em que a pessoa modifica seu estilo de vida, evitando a recaída, atingindo abstinência e consolidando as mudanças. Vale ressaltar que o estágio motivacional do paciente foi estudado e considerado como um fator preditor de efetividade no tratamento (DiClemente \& Prochaska, 1998).

A Entrevista Motivacional Breve, também conhecida como Motivational Enhancement Therapy (MET), foi desenhada para ser uma intervenção breve em intensidade e duração. Ela é mais vantajosa quando aplicada em ambientes com grande demanda de atendimento e pouca disponibilidade de tempo e profissionais. Três importantes revisões examinaram a eficácia das IBs baseadas nos princípios da EM (Noonan \& Moyers, 1997; Dunn et al., 2001; Burke, Arkowitz \& Menchola, 2003) e concluíram que a EM Breve é eficaz para vários problemas comportamentais relacionados ao uso de substância como álcool, maconha e opiáceos (Noonan \& Moyers, 1997); é eficaz quando usada na intensificação de outros tratamentos de abuso de substância, funcionando melhor para o beber problemático e tratamentos intensivos do consumo de substâncias, sendo que o efeito da EM não diminuiu ao longo do tempo (Dunn et al., 2001) e é mais eficaz do que o não-tratamento e tão eficaz quanto qualquer outro tratamento ativo cientificamente reconhecido para o uso de álcool, outras drogas e dieta/exercício (Burke, Arkowitz \& Menchola, 2003).
A primeira revisão metanalítica sobre a eficácia da Entrevista Motivacional Breve exclusivamente para problemas relacionados com o álcool (Vasilaki et al., 2006) comparou 15 ensaios clínicos randomizados para medir sua eficácia comparando-a ao não-tratamento e também a tratamentos semelhantes. Nesta revisão metanalítica, as pesquisas que compararam a EM Breve de duração média de 87 minutos com a ausência de tratamento tiveram resultados estatísticos significantes e superiores a favor da EM Breve na redução do consumo do álcool em usuários nocivos em avaliação de curto prazo ( $\leq 3$ meses). As pesquisas que compararam a EM Breve de 53 minutos com outros tratamentos semelhantes comprovaram que a primeira é mais eficaz do que qualquer outro tipo de intervenção (terapia cognitivocomportamental, aconselhamento diretivo-confrontativo, aconselhamento baseado em habilidades, intervenção educacional). Este estudo também chegou à conclusão de que a EM Breve é eficaz tanto para pacientes que procuram tratamento quanto para aqueles que não procuram. Ao comparar-se um grupo com o outro, a EM Breve se mostrou mais eficaz quando aplicada a usuários nocivos do álcool ou com baixa dependência que procuram pelo tratamento.

O objetivo deste artigo é fazer uma revisão bibliográfica a respeito do uso da Entrevista Motivacional Breve em indivíduos com uso nocivo ou dependência de álcool, utilizando estudos internacionais e nacionais, com vista a discutir a aplicabilidade desta nova abordagem no Brasil.

\section{MÉTODO}

Foi realizada uma busca computadorizada de artigos pelos bancos de dados Medline, Lilacs, Scielo, Cochrane, PsycoInfo, Pubmed, Webscience, cobrindo o período de 1997 a 2007, utilizando-se as palavras-chave "entrevista", "motivacional", "breve", "álcool”, "alcoolismo", ("motivational”, “interviewing", "brief”, “alcohol”, "alcoholism"). Foram considerados artigos originais, nos idiomas português, inglês e espanhol. Foram selecionados os estudos que utilizaram estratégias da Entrevista Motivacional Breve em indivíduos com uso nocivo ou dependência de álcool. Incluíram-se estudos nacionais nesta área que não apareceram na busca eletrônica e excluíram-se da busca estudos que abrangiam outras substâncias químicas (tabaco, cocaína, maconha, etc.) além do álcool, bem como os artigos de revisão sistemática e metanálises.

Com base nestes critérios, foram selecionados e localizados para esta revisão quinze artigos científicos e três teses sobre Entrevista Motivacional Breve em 
indivíduos com uso nocivo ou dependência de álcool, perfazendo um total de dezoito publicações.

\section{RESULTADOS}

\section{Características da amostra}

A Tabela 1 apresenta as características dos dezoito estudos identificados nesta revisão quanto à amostra estudada e ao padrão do consumo de álcool.

Tabela 1. Características da Amostra Estudada Pelas Pesquisas Identificadas Nesta Revisão ( $=18)$

\begin{tabular}{lc}
\hline & Frequência \\
\hline População $(\mathbf{n}=\mathbf{1 8})$ & 12 \\
\hline Adultos & 5 \\
Adultos jovens & 1 \\
Adolescentes & \\
\hline Número de Sujeitos $(\mathbf{n}=\mathbf{1 8})$ & 8 \\
\hline$<100$ & 6 \\
100 - 300 & 3 \\
301 - 900 & 1 \\
$>900$ & 3 \\
\hline Sexo $(\mathbf{n}=\mathbf{1 7})$ & 1 \\
\hline Homens & 13 \\
Mulheres & \\
\hline Homens e mulheres & 8 \\
\hline Padrão de consumo $(\mathbf{n}=\mathbf{1 8})$ & 6 \\
\hline Uso nocivo & 4 \\
Dependência & \\
Uso nocivo e dependência & \\
\hline
\end{tabular}

Quanto à população investigada, quatro estudos foram realizados com população estudantil utilizando como critério o diagnóstico de uso nocivo do álcool, sendo três estudos realizados com estudantes universitários (LaBrie, Pedersen, Earleywine \& Olsen, 2006; Carey, Carey, Maisto \& Henson, 2006; Collins, Carey \& Smyth, 2005) e apenas um com adolescentes (Monti et al., 1999). Cinco estudos investigaram uma população com comorbidades físicas ou psiquiátricas, tais como uso de drogas injetáveis (Stein, Charuvastra, Maksad \& Bradley, 2002), portadores de HIV (Aharonovich et al., 2006), pacientes psiquiátricos (Hulse \& Tait, 2002; Graeber, Moyers, Griffith, Guajardo \& Toningan, 2003) e pacientes internados (Saitz et al., 2007; Hulse \& Tait, 2002). A maioria dos estudos se deu em população adulta que não procurou por tratamento e cuja idade média situava-se entre 31,7 e 46,4 anos. Quase todas as pesquisas trabalharam com ambos os sexos, com exceção de Kelly, Halford e Young (2000), Oliveira (2001), Leontieva et al. (2005), LaBrie et al. (2006), que estudaram apenas um gênero, e Jaeger (2003), que não relatou o sexo dos entrevistados.

Quanto ao padrão de consumo de álcool, cinco pesquisas testaram a intervenção em população cuja maioria (70 a $80 \%)$ era de usuários de álcool com dependência grave (Alwyn, John, Hodgson \& Phillips, 2004; Jaeger, 2003; Oliveira, 2001; Moraes, 2007, Graeber et al., 2003). Foram realizados 08 estudos em usuários com uso nocivo de álcool (Hungerford et al., 2003; Monti et al., 1999; LaBrie et al., 2006; Aharonovich et al., 2006; Bazargan-Hejazi et al., 2005; Carey et al., 2006; Collins et al., 2005; Leontieva et al., 2005). Os outros estudos tiveram participantes com padrões de consumo que variaram desde uso nocivo até vários níveis de dependência.

\section{Aplicabilidade e eficácia da Entrevista Motivacional Breve}

A Tabela 2 apresenta dados sobre como foi aplicada a EM Breve nos 18 estudos identificados nesta revisão, bem como sua eficácia.

Tabela 2. Características da Entrevista Motivacional Breve $(\mathrm{EMB})(\mathrm{N}=18)$

\begin{tabular}{|c|c|}
\hline & Frequência \\
\hline \multicolumn{2}{|l|}{ Intervenção (n = 19) } \\
\hline EMB Sozinha & 15 \\
\hline EMB + outras abordagens & 4 \\
\hline \multicolumn{2}{|l|}{ Número de sessões $(n=17)$} \\
\hline 1 & 9 \\
\hline 2 & 1 \\
\hline 3 & 1 \\
\hline 4 & 4 \\
\hline$\geq 5$ & 2 \\
\hline \multicolumn{2}{|l|}{ Tempo total da intervenção/ minutos $(n=13)$} \\
\hline$<20$ & 1 \\
\hline $20-40$ & 5 \\
\hline $41-60$ & 1 \\
\hline $120-240$ & 4 \\
\hline 360 & 2 \\
\hline \multicolumn{2}{|l|}{ Intervencionista $(\mathrm{n}=17)$} \\
\hline Profissional especializado em dependência & 6 \\
\hline Psicólogo & 5 \\
\hline Psicólogo e outros profissionais da saúde & 3 \\
\hline Outros profissionais da saúde & 3 \\
\hline \multicolumn{2}{|l|}{ Componentes da EMB (respostas múltiplas) } \\
\hline Feedback personalizado & 10 \\
\hline Prós e contras/ Balança decisional & 10 \\
\hline Conscientização sobre o consumo & 7 \\
\hline Metas/ plano de ação & 9 \\
\hline Prontidão para mudança & 2 \\
\hline \multicolumn{2}{|l|}{ Resultado da EMB (n = 19) } \\
\hline EMB Eficaz & 3 \\
\hline EMB >Controle & 14 \\
\hline $\mathrm{EMB}=$ Controle & 2 \\
\hline
\end{tabular}


Quanto ao tipo de intervenção, em treze pesquisas a EM Breve foi oferecida sozinha e, na maioria das vezes, comparada a um tratamento usual ou nãotratamento. Somente em 04 pesquisas a EM foi comparada a outro tipo de intervenção (Hulse \& Tait, 2002; Graeber et al., 2003; Collins et al., 2005; Sellman et al., 2001). Em 05 pesquisas a EM foi oferecida juntamente com outras abordagens (Alwyn et al., 2004; Kelly, Halford \& Young, 2000; Aharonovich et al., 2006; Carey et al. 2006; Sellman et al., 2001). As intervenções foram oferecidas individualmente, com exceção de uma delas, (Jaeger, 2003) que realizou intervenção grupal.

Os profissionais que aplicaram a intervenção foram variados: pesquisadores, psicólogos, enfermeiros, psiquiatras, agentes comunitários, estudantes de psicologia, assistentes sociais, promotores de saúde, sendo que pelo menos $50 \%$ das pesquisas tiveram o psicólogo ou estudante de psicologia como único pesquisador. Na maioria dos estudos houve treinamento específico para a aplicação da EM Breve, com exceção de duas pesquisas (Graeber et al., 2003, Collins et al., 2005).

O tempo de aplicação da intervenção variou entre uma e seis sessões, com tempo total de duração entre dez e 360 minutos. Em uma pesquisa não houve interação pessoal, pois a intervenção ocorreu por intermédio de carta (Collins et al., 2005).

Quanto ao local da pesquisa, pôde-se observar que a EM Breve foi aplicada com sucesso em lugares variados, como, enfermarias de hospitais, prontossocorros, domicílio do entrevistado, ambulatórios especializados e centros de pesquisas.

Os principais componentes da EM breve oferecidos nas intervenções foram o feedback personalizado, prós e contra e/ou balança decisional, conscientização sobre o consumo de álcool com seus riscos e consequências e a elaboração de um plano de mudança.

Quanto aos estudos de seguimentos, quase todas as pesquisas realizaram avaliação nos meses ao término da intervenção (Hungerford et al., 2003; Monti et al., 1999; Alwyn, John, Hodgson \& Phillips, 2004; LaBrie, Pedersen, Earleywine \& Olsen, 2006; Kelly, Halford \& Young, 2000; Stein, Charuvastra, Maksad \& Bradley, 2002; Jaeger, 2003; Oliveira, 2001; Aharonovich et al., 2006; Bazargan-Hejazi et al., 2005; Hulse \& Tait, 2002; Graeber et al., 2003; Carey et al. 2006; Sellman et al., 2001; Saitz et al., 2007; Leontieva et al., 2005), e por este intermédio puderam comprovar que a EM Breve teve sua eficácia mantida por vários meses. Geralmente os seguimentos foram conduzidos aos três, seis, e doze meses.
Quanto à eficácia da intervenção, a EM Breve se mostrou mais eficaz quando comparada a tratamentos usuais e a outras modalidades de tratamento, e eficaz quando comparada a nenhum tratamento. Os principais indicadores de sucesso da intervenção foram: diminuição do consumo álcool por dia/semana e por episódio, redução dos danos relacionados ao álcool, aderência ao tratamento, dias de abstinência e prontidão para mudança. Somente duas pesquisas não obtiveram resultados favoráveis à EM (Jaeger, 2003; Saitz et al., 2007).

\section{Pesquisas nacionais}

Foram identificados três estudos brasileiros que ofereceram abordagem semelhante à dos estudos internacionais (Moraes, 2007; Oliveira, 2001; Jaeger, 2003). Nos três estudos foi administrada a EM Breve em quatro sessões, a indivíduos dependentes de álcool que já estavam passando por algum tipo de intervenção em relação ao álcool (ambulatorial ou internação hospitalar), e seus resultados foram comparados com os do tratamento convencional ou grupo-controle. Moraes (2007) aplicou a EM Breve através de visitas domiciliares, Oliveira (2001) fez atendimentos ambulatoriais individuais e Jaeger (2003) realizou atendimentos ambulatoriais em grupo.

\section{DISCUSSÃO}

Os resultados desta revisão mostraram que a EM Breve pode ser oferecida a clientes de várias faixas etárias, de ambos os sexos, portadoras ou não de comorbidades físicas ou psiquiátricas, com padrão de consumo desde aquele dos usuários abusivos de álcool até o daqueles cuja dependência já estava estabelecida e que procuraram ou não por tratamento.

As pesquisas analisadas nesta revisão trabalharam com uma população com consumo de álcool variado, incluindo uso nocivo, os vários graus de dependência alcoólica e a considerada dependência severa. Os pesquisadores que buscaram clientes que não procuravam por tratamento conseguiram uma amostra mais jovem e com menor grau de comprometimento em relação ao álcool e suas consequências. Seus resultados foram compatíveis com os de outras pesquisas, que sugerem uma eficácia melhor da EM Breve quando aplicada em indivíduos com baixa dependência (Dunn et al., 2001; Vasilaki et al., 2006). Por outro lado, os pesquisadores que buscaram clientes hospitalizados ou já incluídos em algum programa de tratamento do álcool ou comorbidades, trabalharam com uma população mais velha e com 
maior dependência de álcool e maior comprometimento físico, cognitivo e social. Estudos apontam que estes fatores podem, em algum grau, interferir negativamente nos resultados da EM Breve (Dunn et al., 2001; Vasilaki et al., 2006), mas estes fatores não invalidaram os bons resultados alcançados pela maioria dos estudos aqui apresentados.

\section{A aplicabilidade da EM Breve}

Através desta revisão pudemos observar que a EM Breve pode ser oferecida com sucesso, sozinha ou acoplada a outras técnicas, quer em comparação com o tratamento usual quer com grupo-controle. Uma das pesquisas desta revisão demonstrou que a EM Breve, quando oferecida juntamente com outra técnica, tem uma eficácia maior do que quando oferecida isoladamente (Carey et al., 2006).

Os dois estudos desta revisão que não obtiveram resultados favoráveis em favor da EM Breve foram realizados com população internada ou com alta recente, com média de idade acima de 44 anos e dependência grave (Jaeger, 2003; Saitz et al., 2007). Por outro lado, outros estudos também trabalharam com uma população dependente de álcool de forma preponderantemente grave, com comorbidades e originária de tratamentos para dependência química ou internação hospitalar, e obtiveram resultados estatisticamente superiores aos do tratamento usual ou de controle (Oliveira, 2001; Moraes, 2007; Hulse \& Tait, 2002; Graeber et al., 2003; Alwyn et al., 2004).

As avaliações de seguimento mostraram que a EM Breve se sustenta por períodos de seis a doze meses, mas não se conhecem os fatores específicos que contribuem para que estes dados sejam sustentados. Hulse e Tait (2003) fizeram uma avaliação de seguimento cinco anos após a intervenção (Hulse \& Tait, 2002) e descobriram que o efeito da EM Breve se perdeu ao longo deste tempo, ficando igual ao índice da outra intervenção comparada no estudo.

Esta revisão bibliográfica mostrou que a EM Breve pode ser aplicada com sucesso por qualquer profissional da saúde, seja este estudante, graduado ou pós-graduado, desde que receba treinamento específico para esta abordagem. Observamos que em duas pesquisas não houve treinamento específico da equipe (Graeber et al., 2003; Collins et al., 2005). Nestes casos, quem ofereceu a intervenção foram os próprios pesquisadores, que já tinham formação e experiência prévia com a técnica e os princípios da EM.

O tempo total da intervenção teve uma ampla variação - de uma a seis sessões e de dez a 360 minutos. Collins et al. (2005) ofereceram a EM Breve através de uma carta com feedback personalizado, e esta intervenção foi mais eficaz do que uma orientação-padrão sobre o álcool oferecido também por carta. A eficácia com um tempo de intervenção tão curto como 20 minutos, é confirmada pelo maior estudo em EM Breve já realizado, o Project MATCH (1997), o qual concluiu que tratamentos breves - entre uma e quatro sessões motivacionais - podem produzir resultados comparáveis aos de tratamentos intensivos e de longa duração.

Para encontrar usuários nocivos ou dependentes de álcool e intervir precocemente, os pesquisadores realizaram rastreamento em ambientes bastante variados, como escolas, universidades, prontossocorros, enfermarias de hospitais, ambulatórios de outras especialidades, ou divulgaram e ofereceram seus serviços em sites especializados e na mídia, além de clínicas de tratamento de álcool e drogas, evidenciando a ampla variedade de possibilidades de atuação desta abordagem. Isso mostra que é possível encontrar e intervir na população que não busca por tratamento e muitas vezes não tem consciência de estar fazendo uso nocivo de álcool e sofrendo as consequências deste abuso. A partir da intervenção precoce, aquelas pessoas passaram a ter consciência do seu consumo e dos riscos e consequências do abuso de álcool e, na maioria dos casos, puderam reduzir este consumo e sair da zona de risco, ou mesmo buscar um tratamento especializado. Profissionais de saúde que desejam se empenhar na prevenção de problemas relacionados ao álcool não precisam se limitar ao espaço físico do consultório, nem mesmo esperar que o paciente venha até ele. Lugares com grande circulação de pessoas, como os prontossocorros, foram adequados para o usuário de álcool ser abordado e receber informações e aconselhamento sobre o consumo de bebidas alcoólicas (Hungerford et al., 2003).

Quanto aos componentes da EM oferecidos, pôdese observar que alguns elementos estavam sempre presentes na intervenção, como feedback personalizado, conscientização sobre o consumo (consumo de risco e suas consequências), avaliação dos prós e contras de diminuir/parar ou continuar com o consumo e a elaboração de um plano de ação. Estes são procedimentos da $E M$ rápidos e de fácil administração e podem ter contribuído para a eficácia das pesquisas analisadas nesta revisão. LaBrie et al. (2006) pesquisaram apenas um componente da EM (a Balança Decisional) comprovaram que administrar somente este exercício de prós e contras a intervenção foi mais eficaz do que o grupo controle. Leontieva et 
al. (2005) utilizaram a escala de prontidão para mudança para avaliar se ela tem valor preditivo na mudança do comportamento de beber problemático, e tiveram resultados positivos. Vários pesquisadores sugerem outros estudos que possam medir quais componentes da EM são responsáveis pelo sucesso da intervenção e para a manutenção de seus efeitos positivos a médio e longo prazo.

\section{Pesquisas nacionais sobre a EM Breve em indivíduos com dependência de álcool}

Os estudos brasileiros tiveram amostras de pacientes com dependência grave maiores, se comparados aos estudos internacionais. Em apenas uma das pesquisas brasileiras (Jaeger, 2003) a EM Breve foi oferecida em grupo e não houve uma eficácia significativamente maior a favor da intervenção. Apesar de existirem modelos de EM em grupo (Ingersoll, Wagner \& Gharib, 2002; Jaeger \& Oliveira, 2003), há poucos estudos controlados sobre esta intervenção na população eleita por esta revisão. Pesquisadores preconizam que a EM em grupo pode não ser efetiva para todos os pacientes, estando a sua efetividade relacionada a algumas características da população estudada. Não se sabe ainda que características sejam estas, podendo ser desde a existência de comorbidades psiquiátricas até mesmo a gravidade da dependência.

No Brasil ainda existem poucas pesquisas acerca do uso da EM Breve em usuários de álcool. Profissionais da saúde de todas as áreas devem ser encorajados a conhecer mais sobre a EM, para atuar em indivíduos que começam a apresentar um padrão nocivo de uso do álcool ou já são dependentes e avaliar esta prática através da pesquisa científica para que possamos aplicar esta abordagem de forma disseminada e com o mesmo know how dos cientistas estrangeiros. Mais pesquisas são necessárias para que se encontrem meios eficientes, eficazes e de baixo custo para tratar estes indivíduos que se encontrem nos diferentes estágios desta doença.

\section{CONCLUSÃO}

A Entrevista Motivacional Breve tem se mostrado uma intervenção efetiva para reduzir o consumo do álcool e aumentar a motivação para a mudança do padrão de beber, bem como aumentar a procura e a adesão de usuários de álcool a um tratamento formal e especializado para a dependência alcoólica. É uma intervenção de baixo custo e facilmente aplicável em qualquer ambiente de saúde ou na comunidade.
As autoras apontam a necessidade de estudos complementares nesta área, bem como a implementação desta técnica nos serviços de atenção primária da saúde e como abordagem inicial no tratamento da dependência.

\section{REFERÊNCIAS}

Aharonovich, E.; Hatzenbuehler, M. L.; Johnston, B.; O’Leary, A.; Morgenstern, J. et al (2006). A low-cost, sustainable intervention for drinking reduction in the HIV primary care setting. AIDS Care, 18(6), 561-8.

Alwyn, T.; John, B.; Hodgson, R. J., \& Phillips, C. J. (2004). The addition of a psychological intervention to a home detoxification programme. Alcohol Alcohol, 39(6), 536-41.

Bazargan-Hejazi, S.; Bing, E.; Bazargan, M.; Der-Martirosian, C.; Hardin, E. et al (2005). Evaluation of a brief intervention in an inner-city emergency department. Annals of Emergency Medicine, 46(1), 67-76.

Burke, B.L.; Arkowitz, H., \& Menchola, M. (2003).The efficacy of motivational interviewing: a meta-analysis of controlled clinical trials. Journal of Consulting and Clinical Psychology, 71, 784861.

Carey, K. B.; Carey, M. P.; Maisto, S. A., \& Henson, J.M. (2006). Brief motivational interventions for heavy college drinkers: a randomized controlled trial. Journal of Consulting and Clinical Psychology, 74(5), 943-954.

Collins, S. E.; Carey, K. B., \& Smyth, J. (2005). Relationships of linguistic and motivations variables with drinking outcomes following two mailed brief interventions. Journal of Studies on Alcohol, 66(4), 526-35.

DiClemente, C. C., Prochaska, J. O. (1998). Toward a comprehensive, transtheoretical modelo $\mathrm{f}$ change: Stages of change and addictive behaviors. Em: Miller, W. R.; Heather, N.; (Orgs) Treating addictive Behaviors (pp. 3-24). New York: Plenum Press.

Dunn, C.; Deroo, L., \& Rivara, F. P. (2001). The use of brief interventions adapted from motivational interviewing across behavioral domains: a systematic review. Addiction, 96(12), 1725-42.

Figlie, N. B.; Pillon, S. C.; Laranjeira, R., \& Dunn, J. (2000). The frequency of smoking and problem drinking among general hospital inpatients in Brazil - using the AUDIT and Fagerström questionnaires. São Paulo Medical Journal, 118(5):139-43.

Fleming, M. F., \& Manwell, L. B. (1999). Brief Intervention in primary care setting. Alcohol Research \& Health, 128-137.

Graeber, D. A.; Moyers, T. B.; Griffith, G.; Guajardo, E., \& Tonigan, S. (2003). A pilot study comparing Motivational Interviewing and educational intervention in patients with schizophrenia and alcohol use disorders. Community Mental Health Journal, 39(3), 189-202.

Hettema, J.; Steele, J., \& Miller, W. R. (2005). Motivational Interviewing. Annual Review of Clinical Psychology, 1, 91111.

Hulse, G. K., \& Tait, R. J. (2003). Five-year outcomes of a brief alcohol intervention for adult in-patients with psychiatric disorders. Addiction, 98(8), 1061-8. 
Hulse, G. K. \& Tait, R. J. (2002). Six-month outcomes associated with a brief alcohol intervention for adult in-patients with psychiatric disorders. Drug Alcohol Review, 21(2), 105-12.

Hungerford, D. W. \& Williams, J. M.; Furbee, P. M.; Manley, W. G.; Helmkamp, J. C.; Horn, K.; Pollock, D. A. (2003). Feasibility of screening and intervention for alcohol problems among young adults in the ED. American Journal of Emergency Medicine, 2l(1), 14-22.

Ingersoll, K. S.; Wagner, C. C. \& Gharib, S. (2002). Motivational Groups for Community Substance Abuse Programs. (2 $2^{\mathrm{a}}$ edição) Richmond: Mid-Atlantic ATTC.

Jaeger, A. \& Oliveira, M. S. (2003). Entrevista Motivacional em Grupos: uma proposta terapêutica breve para o tratamento da dependência química. Boletim de Psicologia, 53(118), 25-34.

Jaeger A. (2003). A Entrevista Motivacional em grupo no tratamento de alcoolistas. Dissertação de Mestrado, Pontifícia Universidade Católica do Rio grande do Sul, Porto Alegre .

Kelly, A. B.; Halford, W. K. \& Young, R. M. (2000). Maritally distressed woman with alcohol problems: the impact of a shortterm alcohol-focused intervention on drinking behavior and marital satisfaction. Addiction, 95(10), 1537-49.

LaBrie, J. W.; Pedersen, E. R.; Earleywine, M. \& Olsen, H. (2006). Reducing heavy drinking in college males with the decisional balance: analyzing an element of Motivational Interviewing. Addictive Behaviors, 31 (2), 254-63.

Laranjeira, R.; Pinsky, I.; Zaleski, M. \& Caetano, R. (2007). I Levantamento Nacional sobre os padrões de consumo de álcool na população brasileira. Brasília: SENAD.

Leontieva, L.; Horn, K.; Haque, A.; Helmkamp, J.; Ehrlich, P. \& Williams, J. (2005). Readiness to change problematic drinking assessed in the emergency department as a predictor of change. Journal of critical care, 20(3), 251-256.

Miller, W. R. \& Rollnick, S. (1991). Motivational Interviewing: preparing people to change addictive behaviour. New York: The Guilford Press.

Monti, P. M.; Colby, S. M.; Barnett, N. P.; Spirito, A.; Rohsenow, D. J.et al (1999). Brief intervention for harm reduction with alcohol positive older adolescents in a hospital emergency department. Journal of Consulting and Clinical Psychology, 67(6), 989-94.

Moraes, E. (2007). Visita domiciliar: avaliação do impacto clínico e econômico em um tratamento ambulatorial para dependentes de álcool. Dissertação de Mestrado. UNIFESP/Escola Paulista de Medicina, São Paulo.

Noonan, W. C., \& Moyers, T. B. (1997). Motivational interviewing: a review. Journal of Substance Misuse, 2, 8-16.

Oliveira, M. S. (2001). Eficácia da intervenção motivacional em dependentes do álcool. Dissertação de Doutorado. UNIFESP/Escola Paulista de Medicina, São Paulo.

Project MATCH Research Group. (1997). Matching alcoholism treatments to client heterogeneity: Project MATCH pos treatment drinking outcomes. Journal of Studies on Alcohol, 58, 7-29.

Rubak, S.; Sandboek, A.; Lauritzen, T., \& Christensen, B. (2005). Motivational interviewing: a systematic review and meta-analysis. British Journal of General Practice, 55, 305-312.

Saitz, R.; Palfai, T. P.; Cheng, D. M.; Horton, N. J.; Freedner, N. et al (2007). Brief intervention for medical inpatients with unhealthy alcohol use: a randomized, controlled trial. Annals of Internal Medicine, 146(3), 167-76.

Sellman, J. D.; Sullivan, P. F.; Dore, G. M.; Adamson, S. J., \& McEwan, I. (2001). A randomized controlled trial of motivational enhancement therapy (MET) for mild to moderate alcohol dependence. Journal of Studies on Alcohol, 62(3), 389-96.

Stein, M. D.; Charuvastra, A.; Maksad, J., \& Bradley, J.A. (2002). A randomized trial of a brief alcohol intervention for needle exchangers (BRAINE). Addiction, 97 (6), 691-700.

Vasilaki, E. I.; Hosier, S. G., \& Cox, M. (2006). The efficacy of motivational interviewing as a brief intervention for excessive drinking: a meta-analytic review. Alcohol \& Alcoholism, 41 (3), 328-335.

Recebido em 28/09/2007 Aceito em 23/07/2008

Endereço para correspondência : Cristiane Martins Baía Sales. Rua Botucatu, no 394, Vila Clementino, CEP 04023-061, São

Paulo-SP, Brasil.E-mail: cristianebsales@uol.com.br 